\title{
Differentially expressed and activated proteins associated with non small cell lung cancer tissues
}

E. Nigro ${ }^{1}$, E. Imperlini ${ }^{2,8}$, O. Scudiero ${ }^{1,3}$, M.L. Monaco ${ }^{1}$, R. Polito ${ }^{1}$, G. Mazzarella ${ }^{4}$, S. Orrù ${ }^{1,5}$, A. Bianco ${ }^{6}$ and A. Daniele 1,7 $^{*}$

\begin{abstract}
Background: Lung cancer is a leading cause of mortality. The most common cancer subtype, non small cell lung cancer (NSCLC), accounts for 85-90\% all cases and is mainly caused by environmental and genetic factors. Mechanisms involved in lung carcinogenesis include deregulation of several kinases and molecular pathways affecting cell proliferation, apoptosis and differentiation. Despite advances in lung cancer detection, diagnosis and staging, survival rate still remains poor and novel biomarkers for both diagnosis and therapy need to be identified. In the present study, we have explored the potential of novel specific biomarkers in the diagnosis of NSCLC, and the over-expression/activation of several kinases involved in disease development and progression.

Method: Lung tumor tissue specimens and adjacent cancer-free tissues from 8 NSCLC patients undergoing surgery were collected. The differential activation status of ERK1/2, AKT and IKBa/NF-K $\beta$ was analyzed. Subsequently, protein expression profile of NSCLC vs normal surrounding tissue was compared by a proteomic approach using LC-MS MS. Subsequently, MS/MS outputs were analyzed by the Protein Discoverer platform for label-free quantitation analysis. Finally, results were confirmed by western blotting analysis.

Results: This study confirms the involvement of ERK1/2, AKT, IKBa and NF-K $\beta$ proteins in NSCLC demonstrating a significant over-activation of all tested proteins. Furthermore, we found significant differential expression of 20 proteins $\left(R_{s c} \geq 1.50\right.$ or $\left.\leq-1.50\right)$ of which 7 are under-expressed and 13 over-expressed in NSCLC lung tissues. Finally, we validated, by western blotting, the two most under-expressed NSCLC tissue proteins, carbonic anhydrase I and II isoforms.

Conclusion: Our data further support the possibility of developing both diagnostic tests and innovative targeted therapy in NSCLC. In addition to selective inhibitors of ERK1/2, AKT, IKBa and NF-K $\beta$, as therapeutic options, our data, for the first time, indicates carbonic anhydrase I and II as attractive targets for development of diagnostic tools enabling selection of patients for a more specific therapy in NSCLC.
\end{abstract}

Keywords: Lung cancer, Non small cell lung cancer [NSCLC], ERK1/2, AKT, IKBa, NF-Kß, Carbonic anhydrase I and II isoforms [CAl, II]

\section{Introduction}

Lung cancer (LC) remains the leading cause of cancer death worldwide accounting for 14.1 million new cancer cases and 8.2 million deaths per year $[1,2]$. Worldwide incidence appears to be variable but equally distributed with increasing trends among males and females $[1,2]$. LC is

\footnotetext{
* Correspondence: aurora.daniele@unina2.it

${ }^{1}$ CEINGE-Biotecnologie Avanzate Scarl, Via G. Salvatore 486, 80145 Naples, Italy ${ }^{7}$ Dipartimento di Scienze e Tecnologie Ambientali Biologiche Farmaceutiche, Seconda Università degli Studi di Napoli, Via G. Vivaldi 42, 81100 Caserta, Italy Full list of author information is available at the end of the article
}

stratified into two major subtypes, small cell lung cancer (SCLC) and non-small cell lung cancer (NSCLC), the latter representing 85-90 \% of all cases of LC [3]. Response to current cytotoxic therapies has reached a plateau in terms of response rate and survival $[4,5]$. The development of molecular profiling technologies to assess DNA, RNA, proteins and metabolites heralds a new era in the understanding of the molecular basis of non-small-cell lung carcinoma (NSCLC) leading to potential advances in management and treatment of lung cancer [6]. Novel molecular markers in

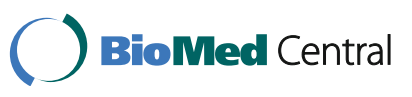


non small-cell lung cancer (NSCLC) also include DNA damage repair genes. The pathogenesis of NSCLC cancer is complex and influenced by multiple factors including: a) environment (i.e. exposure to carcinogens, smoking habit, diet); b) genetic and epigenetic changes (such as p53 and EGFR gene mutations); c) pulmonary and systemic inflammatory conditions (such as concurrent Chronic Obstructive Pulmonary disease) [7-17]. At cellular level, activation status of several kinases involved in cell proliferation, apoptosis and inflammation is central to establishment and development of carcinogenesis. For example, constitutive activation of MEK-ERK, PI3K-AKT and/or PI3K-AKT-NF$\kappa \beta$ pathways plays a key role in oncogenesis and strongly promotes LC invasiveness [13-17]. AKT activation is present in $51 \%$ of NSCLC although AKT mutations are rare $(<1 \%)[18,19]$. However, the role of MEK and PI3K pathways as prognostic and/or predictive markers for cancer remains controversial [20]. Today, the MEK and AKT inhibitors combined with chemotherapy are very promising for the treatment of several human cancers, including NSCLC $[18,19]$.

Despite the introduction of diagnostic tools such as computed tomography (CT) scans, Positrion Emission Tomography (PET) scan and bronchoscopy, the early diagnosis of LC remains unsatisfactory [20]. Circulating biochemical biomarkers such as carcinoembryonic antigen (CEA) TPA, CYFRA 21.1 etc. have a limited impact in early diagnosis whereas methylation-based assays may represent a more promising strategy for early detection and follow-up of NSCLC by means of a differential label-free proteomic analysis [21]. Actually, cancer detection, diagnosis and staging may be further improved by molecular selection $[2,21]$. As current early detection procedures and treatments are unsatisfactory in terms of impact on quality of life and overall survival novel biomarkers need to be identified. In this context, the emerging "-omics" approach represents an important tool in the detection and quantitation of novel keyproteins as putative biomarkers for $\mathrm{LC}[3,22]$.

In this study, we collected lung tumour specimens from 8 NSCLC chemotherapy naive patients undergoing surgery as well as surrounding cancer-free lung tissues and: a) investigated activation status of ERK, AKT, IKB $\alpha$ and NF- $\kappa \beta$; b) defined novel specific biomarkers for NSCLC diagnosis by means of a differential label-free proteomic analysis. We found: a) activation of all tested proteins and b) 20 proteins differentially expressed between NSCLC and controls. Finally, we validated, by western blotting, the two most differentially expressed proteins, carbonic anhydrase I and II (CAI, CAII) isoforms.

\section{Materials and methods} Lung tissues sampling

NSCLC tumour tissue specimens and surrounding cancerfree tissue were collected from eight chemotherapy naïve patients who underwent thoracic surgery. Hystological analysis of tumour tissue specimens was conducted by pathologists at Monaldi Hospital of Naples and confirmed the diagnosis as follows: six adenocarcinoma and two adenosquamous carcinoma; surrounding cancer-free lung specimens showed evidence of airway remodeling and signs of mucociliary dysfunction and alveolar destruction consistent with diagnosis of Chronic Obstructive pulmonary diseases. Samples were immediately frozen at $-80{ }^{\circ} \mathrm{C}$. The study was approved by the local ethics committee and conducted in accordance with ethical principles stated in most recent version of the Declaration of Helsinki on the applicable guidelines for good clinical practices.

\section{Protein extraction}

Lung tissues were homogenized in buffer containing $50 \mathrm{mM}$ Tris-HCl, pH 7.5, $150 \mathrm{mM} \mathrm{NaCl}, 1 \%$ [v/v] Triton X-100, $10 \%$ [v/v] glycerol, $0.5 \mathrm{mM}$ PMSF and complete mini protease inhibitor cocktail (Roche, Basel, Switzerland). Tissues were disrupted using a Dounce homogenizer and centrifuged at $16,000 \mathrm{~g}$ at $4{ }^{\circ} \mathrm{C}$ for $30 \mathrm{~min}$. Protein concentrations were determined using Bradford's reagent (Biorad Laboratories, CA, USA).

\section{Western blotting analysis}

Protein extracts $(30 \mu \mathrm{g})$ from each of 8 NSCLC and cancer-free lung tissue were incubated in Laemmli buffer with DTT, resolved on SDS-PAGE and then transferred onto nitrocellulose membranes (GE Healthcare, TX, USA) by Mini trans-blot electrophoresis transfer as previously described (Bio-Rad Laboratories, CA, USA) [23]. p-ERK1/ 2, ERK1/2, p-AMPK, AMPK, p-AKT, AKT, p-P38, P38, p$\mathrm{IKB} \alpha$, IKB $\alpha$ antibodies were from Cell Signaling, Netherlands; NF- $\kappa \beta$ antibody was from BD bioscience; CAI antibody was from Santa Cruz Biotechnology, MA, USA; CAII antibody was from Rockland, PA, USA; GAPDH and $\beta$-actin antibodies were from Sigma-Aldrich, MO, USA. Immunoblots were detected using the ECLAdvance Western Blotting Detection kit (GE Healthcare, TX, USA). Western blot images were scanned by PDquest 7.1 software (Bio-Rad Laboratories, CA, USA). Densitometric measurements were made with the Quantity One 4.5 tool (Bio-Rad Laboratories, CA, USA). Each experiment was performed at least three times in duplicate.

\section{In-gel digestion}

Pooled protein extracts $(100 \mu \mathrm{g})$ were obtained by mixing equal amounts of all 8 NSCLC samples as well as of corresponding controls. Both pooled protein samples were re-suspended in Laemmli buffer with 0.1 M DTT, incubated at $95{ }^{\circ} \mathrm{C}$ for $5 \mathrm{~min}$ and separated by SDSPAGE. Molecular weight was estimated by using Precision Plus All Blue protein standards (Bio-Rad Laboratories, CA, USA). Protein electrophoretic patterns 
were then visualized using GelCode Blue Stain Reagent. Protein bands of interest were excised from gel lanes, crushed and washed first with acetonitrile (ACN) and then with $50 \mathrm{mM}$ ammonium bicarbonate (AMBIC); enzymatic digestions were carried out as previously described [24]. In summary, protein samples were reduced in $10 \mathrm{mM}$ DTT for $45 \mathrm{~min}$ at $56{ }^{\circ} \mathrm{C}$ and alkylated in $55 \mathrm{mM}$ iodoacetamide in $50 \mathrm{mM}$ AMBIC for $30 \mathrm{~min}$ at RT in the dark. Subsequently, gel particles were washed with $50 \mathrm{mM}$ AMBIC and $\mathrm{ACN}$ and rehydrated in a modified trypsin solution (10 ng/ $\mu \mathrm{l})$ (Sigma, MO, USA) in $50 \mathrm{mM}$ AMBIC $\mathrm{pH} 8.5$, at $4{ }^{\circ} \mathrm{C}$ for $2 \mathrm{~h}$. After removal of enzymatic solution, an aliquot of buffer solution was added and incubated at $37{ }^{\circ} \mathrm{C}$ for $18 \mathrm{~h}$. The supernatant was collected, while gel pieces were subjected to further extraction in $\mathrm{ACN}$ at $37{ }^{\circ} \mathrm{C}$ for $15 \mathrm{~min}$. Peptides obtained from extraction were pooled, vacuum-dried and resuspended in $0.2 \% \mathrm{HCOOH}$ before MS analysis.

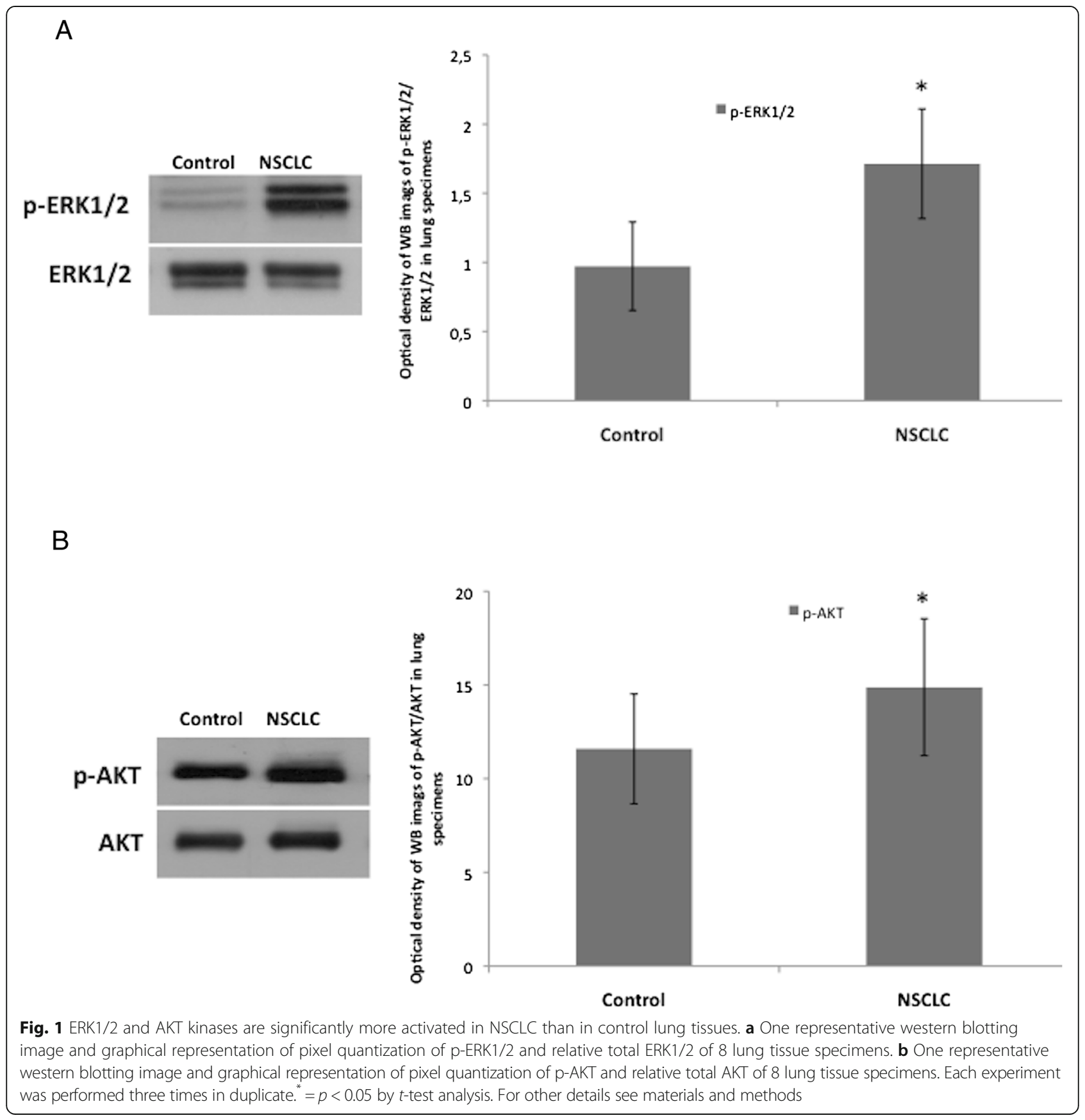




\section{Mass spectrometry analysis}

Peptide mixtures were analyzed by LC-MS MS using the LC/MSD Trap XCT Ultra (Agilent Technologies, CA, USA) equipped with a 1100 HPLC system and a chip cube (Agilent Technologies, CA, USA) as previously described [24]. Briefly, after loading, peptide mixture (8 $\mu \mathrm{l}$ in $0,2 \% \mathrm{HCOOH}$ ) was pre-concentrated, washed at $4 \mu \mathrm{l} / \mathrm{min}$ in $40 \mathrm{nl}$ enrichment column (Agilent Technologies chip) and separated on a RP-C18 column $(75 \mu \mathrm{m} \times 43 \mathrm{~mm})$ at a flow rate of $200 \mathrm{nl} / \mathrm{min}$ with a linear gradient of eluent B ( $2 \%$ formic acid in acetonitryl) in eluent A (2\% formic acid) from 5 to $60 \%$ in $60 \mathrm{~min}$. Peptides were analyzed using data-dependent acquisition of MS scan (400-2000 m/z) followed by MS/MS scans of the three most abundant ions. Dynamic exclusion was used to acquire a more complete survey of the peptides. A permanent exclusion list of the most frequent peptide contaminants was included in the acquisition as previously described [25].

\section{Protein identification and quantitation}

MASCOT software (Matrix Science, London, UK) was used for protein database searching as previously reported
[26]. The searches were performed using the NCBI database and the following standard parameters: Homo Sapiens; one missed cleavage; carboxyamidomethylation of Cys, partial Met oxidation and putative modification of Gln to pyro-Glu, mass tolerance of 300 ppm on precursor ions, and $0.6 \mathrm{Da}$ on the product ions. Individual ion scores $>43$ indicate identity or extensive homology $(p<$ 0.05). For label-free quantitation, Mascot format text files were analyzed by Proteome Discoverer platform (version 1.3; Thermo Scientific, Bremen, Germany), interfaced with an in-house Mascot server (version 2.3, Matrix Science, London, UK). All peptides with FDR $\leq$ 0.01 and a peptide rank of 1 were included. Spectral counts $(\mathrm{SpC})$ were used for estimating protein abundance and comparing the expression of the same protein between tumour and control tissues. SpC log Ratio (Rsc) and Normalized Spectral Abundance Factor (NSAF) were calculated as previously described [23].

\section{Statistical analysis}

Data are expressed as means \pm SD and median. The statistical significance was established at $p<0.05$. Two groups were compared with 2 -tailed unpaired Student $t$-test.
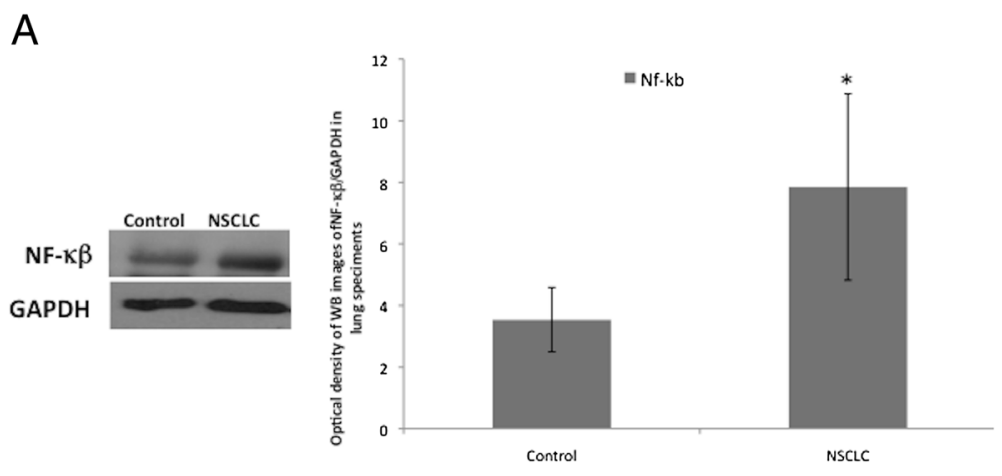

B

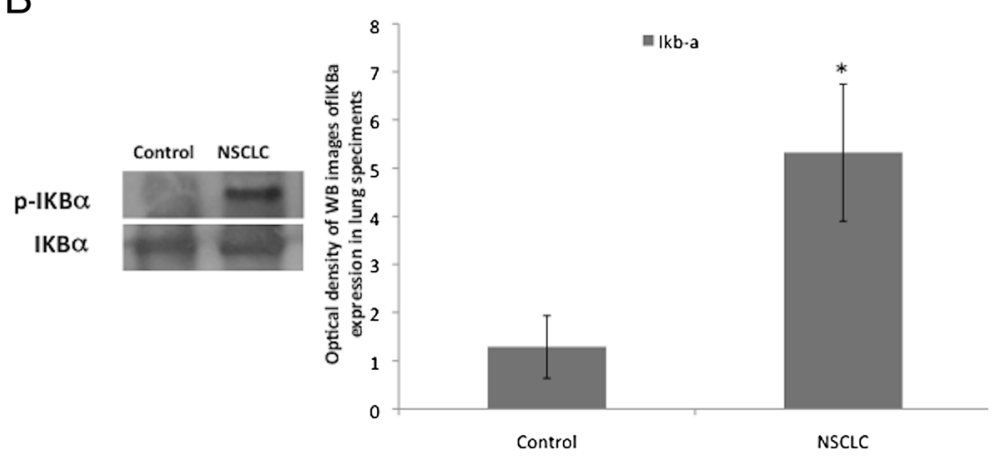

Fig. $2 \mathrm{NF}-\kappa \beta$ and $p-I K B a$ are significantly more expressed in NSCLC than in control lung tissues. a One representative western blotting image and graphical representation of pixel quantization of $\mathrm{NF}-\mathrm{k} \beta$ and relative total GAPDH of 8 lung tissue specimens. $\mathbf{b}$ One representative western blotting image and graphical representation of pixel quantization of $\mathrm{p}-\mathrm{IKBa}$ and total IKBa in 8 lung tissue specimens. Each experiment was performed three times in duplicate. $=p<0.05$ by $t$-test analysis. For other details see materials and methods 


\section{Results}

ERK1/2 and AKT kinases are significantly more activated in NSCLC tissues

To analyze the expression profile of the most relevant proteins regulating cell survival proliferation and apoptosis in NSCLC, we evaluated the activation of MAPK, ERK1/2 and AKT kinases in LC tissue specimens. We demonstrated that $\mathrm{p}$-ERK1/2 and p-AKT activation was statistically different between control cancer-free and NSCLC tissues; in fact, cancer specimens have a significantly higher expression of p-ERK $1 / 2$ and $\mathrm{p}-\mathrm{AKT}$ compared to the control $(p<0.05)$ (Fig. 1a, and $\mathrm{b}$, respectively). In particular, p-ERK1/2 over-activation is 1,8 fold and p-AKT over-activation is 1,3 -fold higher in NSCLC than in cancer-free tissues.

\section{NF-K $\beta$ and IKBa proteins are over expressed/activated in NSCLC tissues}

We analyzed the expression of NF $-\kappa \beta$ and $p-I K B \alpha$, two proteins involved in the control of survival and inflammation and therefore potentially affected in cancer specimens. We found that NSCLC tissues have statistically higher expression of $\mathrm{NF}-\kappa \beta$ and IKB $\alpha$ compared to control cancer-free (Fig. 2a, b, respectively). In particular, $\mathrm{NF}-\kappa \beta$ is over-expressed 2.2 fold while IKB $\alpha$ is 4 -fold over-expressed in NSCLC respect to cancer-free tissues.

\section{Identification of differentially expressed proteins in NSCLC tissues}

We analyzed the protein expression profile of NSCLC and control cancer-free tissues in order to search for potentially LC biomarkers. Pooled protein extracts from 8 NSCLC and those from adjacent cancer-free lung tissues were fractionated onto a $10 \%$ SDS-PAGE. As shown in Fig. 3, electrophoretic patterns between NSCLC and control extracts differed mainly in the low MW gel region. Protein bands from both lanes were excised individually, in-gel digested with trypsin, and analyzed by LC-MS/MS. We identified 38 proteins (see Table 1).

In order to quantitatively compare the protein expression profiles of NSCLC and cancer-free lung tissues, MS/MS outputs were analyzed by the Protein Discoverer platform and submitted to label-free quantitation analysis. Table 2 contains the details of the label-free quantitation method based on spectral counting for protein abundance estimation. In addition to the Normalized Spectral Abundance Factor (NSAF) for each samples, it has been calculated the semi-quantitative parameter $\mathrm{R}_{\mathrm{sc}}$, representing the $\log _{2}$ ratio between the protein expression level of control vs NSCLC lung tissues. This label-free procedure revealed 20 differentially expressed proteins with $R_{s c} \geq 1.50$ or $\leq-1.50$. In Table 2 such species are ranked from the highest $R_{\mathrm{sc}}$ value to the lowest. In particular, our analysis demonstrated that seven proteins with $\mathrm{R}_{\mathrm{sc}} \geq 1.50$ are over-expressed in control, while 13 proteins $R_{\mathrm{sc}} \leq-1.50$ are over-expressed in NSCLC tissues.

\section{Validation of carbonic anhydrase I and II isoforms}

Among proteins identified by MS analysis, we considered and validated carbonic anhydrase I (CAI) and II (CAII) isoforms. In fact, the two isoforms were identified in an intense protein band (MW between $25 \mathrm{kDa}$ and $37 \mathrm{kDa}$ ), present in control and almost absent in the NSCLC (Fig. 3, as indicated by arrow); they were then quantified by label-free quantitation analysis. This procedure confirmed the strong over-expression of CAI and CAII $\left(R_{s c}=2,10\right.$ and $R_{s c}=3,10$, respectively) in the control compared to NSCLC tissues (see Table 2). Western blotting analysis verified the significant under-expression of CAI and CAII proteins in NSCLC tissues compared to the control (Fig. 4a, b).

\section{Discussion}

Surgical resection, when indicated, remains the best treatment option for LC patient whilst radiotherapy and chemotherapy, although effective, have plateaued in terms of response and survival $[18,19]$. This highlights the necessity for earlier diagnosis and more specific therapies to be found. We focused on NSCLC, the most common LC subtype, investigating: a) the activation/

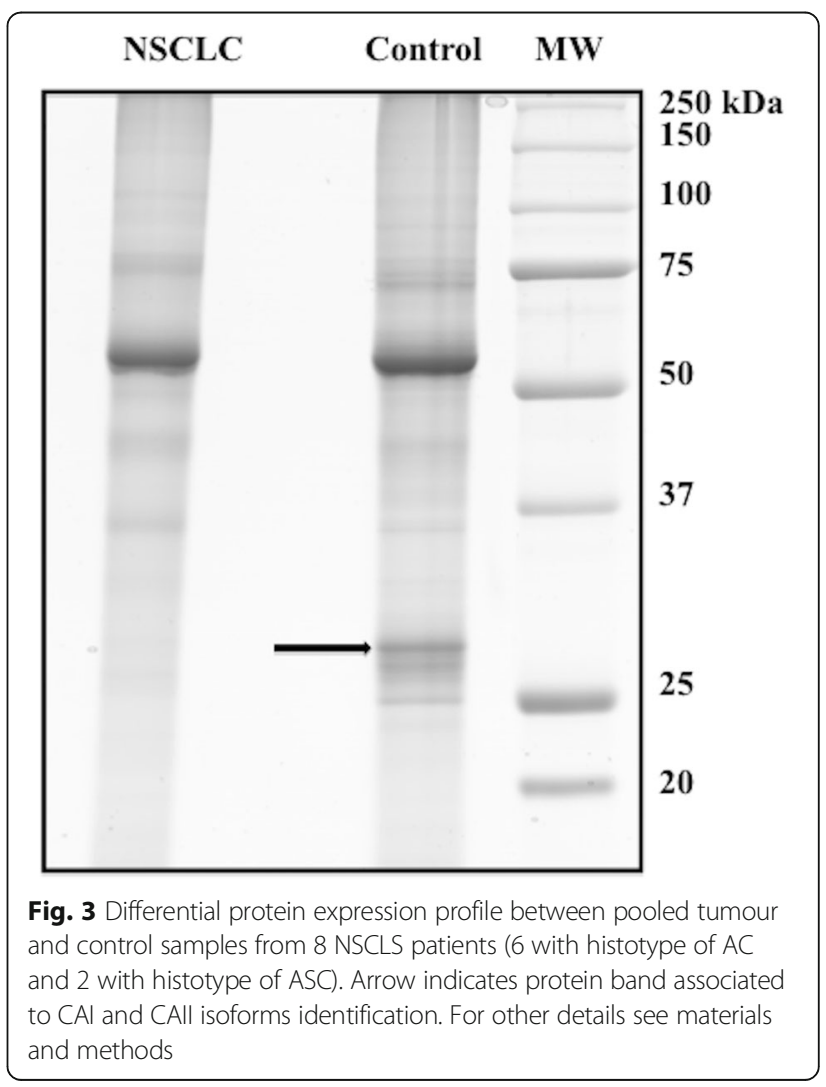


Table 1 Identified proteins in control and NSCLC tissues by MS analysis

\begin{tabular}{|c|c|c|c|c|c|}
\hline \multirow[t]{3}{*}{ Protein } & \multirow[t]{3}{*}{ Gene } & \multirow[t]{3}{*}{ Gene ID } & \multirow[t]{3}{*}{$\mathrm{MW}^{\mathrm{a}}$} & \multirow{3}{*}{$\begin{array}{l}\text { Mascot Score }^{b} \\
{\text { Peptide }]^{c}}^{\text {NSCLC }}\end{array}$} & \multirow{3}{*}{$\begin{array}{l}\text { Mascot Score } \\
{\text { [Peptide }]^{c}}^{\text {Control }} \\
\text { Con }\end{array}$} \\
\hline & & & & & \\
\hline & & & & & \\
\hline lactoferrin precursor & LTF & 12083188 & 80214 & $946[15]$ & $298[5]$ \\
\hline transferrin & TF & 37747855 & 79310 & $581[9]$ & $940[15]$ \\
\hline 78 kDa glucose-regulated protein precursor & HSPA5 & 16507237 & 72402 & $507[8]$ & $503[8]$ \\
\hline moesin & MSN & 4505257 & 67892 & $412[8]$ & $396[7]$ \\
\hline myeloperoxidase, isoform CRA_d & MPO & 119614879 & 76923 & $331[6]$ & $73[1]$ \\
\hline protein disulfide-isomerase A4 precursor & PDIA4 & 4758304 & 73229 & $191[3]$ & $128[2]$ \\
\hline glyceraldehyde-3-phosphate dehydrogenase & GAPDH & 31645 & 36201 & $581[9]$ & $375[6]$ \\
\hline annexin $\mathrm{A} 2$ isoform 2 & ANXA2 & 18645167 & 38808 & $975[16]$ & $504[10]$ \\
\hline annexin A1 & ANXA1 & 4502101 & 38918 & $587[8]$ & $522[7]$ \\
\hline L-lactate dehydrogenase A isoform 1 & LDHA & 5031857 & 36950 & $391[7]$ & N. D. ${ }^{d}$ \\
\hline aldolase A & ALDOA & 28614 & 39706 & $240[4]$ & $65[1]$ \\
\hline peroxiredoxin-1 & PRDX1 & 4505591 & 22324 & $158[3]$ & $253[5]$ \\
\hline Aldo-keto reductase family 1 member C4 & AKR1C4 & 308153631 & 37442 & $155[3]$ & N. D. ${ }^{d}$ \\
\hline 605 acidic ribosomal protein P0 & RPLPO & 4506667 & 34423 & $133[2]$ & N. D. ${ }^{d}$ \\
\hline Actin, beta & ACTB & 16359158 & 42078 & $103[2]$ & $238[4]$ \\
\hline alcohol dehydrogenase beta subunit & $\mathrm{ADH} 1 \mathrm{~B}$ & 178098 & 40665 & N. D. ${ }^{d}$ & $107[2]$ \\
\hline cathepsin D & CTSD & 157879206 & 26511 & $151[3]$ & $131[2]$ \\
\hline stomatin & STOM & 14715077 & 31958 & 112 [2] & N. D. ${ }^{d}$ \\
\hline apolipoprotein A-I, isoform CRA_b & APOA1 & 177827 & 23454 & $566[9]$ & $1057[17]$ \\
\hline ribosomal protein L7 & RPL7 & 1335288 & 29907 & $170[2]$ & $75[1]$ \\
\hline $\begin{array}{l}\text { tyrosine 3-monooxygenase/tryptophan } \\
\text { 5-monooxygenase activation protein, gamma }\end{array}$ & YWHAG & 380765197 & 28373 & $169[2]$ & $96[1]$ \\
\hline $\begin{array}{l}\text { tyrosine 3-monooxygenase/tryptophan 5-monooxygenase } \\
\text { activation protein, beta polypeptide variant }\end{array}$ & YWHAB & 62089104 & 22563 & 48 [1] & $113[2]$ \\
\hline triosephosphate isomerase 1 & TPI1 & 136066 & 26894 & $633[10]$ & $394[6]$ \\
\hline proteasome subunit alpha type- 6 & PSMA6 & 8394076 & 27838 & $184[3]$ & $65[1]$ \\
\hline carbonic anhydrase 1 & CA1 & 4502517 & 28909 & $104[2]$ & $482[8]$ \\
\hline carbonic anhydrase 2 & CA2 & 15080386 & 29215 & N. D..$^{d}$ & $237[4]$ \\
\hline azurocidin & AZU1 & 28977 & 27093 & $130[2]$ & N. D. ${ }^{d}$ \\
\hline phosphoglycerate mutase 1 & PGAM1 & 4505753 & 28900 & $91[2]$ & N. D. ${ }^{d}$ \\
\hline enoyl-CoA hydratase & EHHADH & 1922287 & 31807 & $107[2]$ & N. D. ${ }^{d}$ \\
\hline profilin-1 & PFN1 & 4826898 & 15216 & N. D. ${ }^{d}$ & $103[2]$ \\
\hline endoplasmic reticulum protein 29, isoform CRA_b & ERP29 & 119618398 & 29638 & $102[2]$ & N. D. ${ }^{d}$ \\
\hline pre-serum amyloid $\mathrm{P}$ component & APCS & 337758 & 25495 & $337[5]$ & $318[5]$ \\
\hline peroxiredoxin-6 & PRDX6 & 134254698 & 25011 & $342[6]$ & $299[5]$ \\
\hline MHC class II antigen & HLA-DRB1 & 10185082 & 30525 & N. D..$^{d}$ & $183[3]$ \\
\hline MHC class I antigen & HLA-DQB & 325656872 & 21947 & N. D..$^{d}$ & $150[3]$ \\
\hline Rab5c-like protein & RAB5C & 508285 & 23781 & $101[2]$ & $97[2]$ \\
\hline proteasome subunit beta type- 4 & PSMB4 & 22538467 & 29242 & N. D..$^{d}$ & $211[3]$ \\
\hline rho GDP-dissociation inhibitor & ARHGDIB & 56676393 & 23031 & N. D. ${ }^{d}$ & $122[2]$ \\
\hline
\end{tabular}

${ }^{\mathrm{a}}$ Theoretical MW
bProtein score assigned by Mascot software is derived from ion scores that are $-10^{*}$ Log [p], where $\mathrm{p}$ is the probability that the observed match is a random event

'Number of peptides matched

${ }^{\mathrm{d}}$ Not determined 
Table 2 Spectral counting and protein ratios for differentially expressed proteins

\begin{tabular}{|c|c|c|c|c|}
\hline \multirow[t]{2}{*}{ Protein } & \multirow[t]{2}{*}{ Gene } & \multirow{2}{*}{$\begin{array}{l}\mathrm{NSAF}^{\mathrm{a}} \\
\mathrm{NSCLC}\end{array}$} & $N^{\prime} A F^{a}$ & \multirow[t]{2}{*}{$R_{s c}^{b}$} \\
\hline & & & Control & \\
\hline carbonic anhydrase 2 & CA2 & 0 & 0,00693 & 3,10 \\
\hline MHC class I antigen & HLA-DQB & 0 & 0,0131 & 2,43 \\
\hline alcohol dehydrogenase beta subunit & $\mathrm{ADH} 1 \mathrm{~B}$ & 0 & 0,00356 & 2,43 \\
\hline carbonic anhydrase 1 & CA1 & 0,00559 & 0,0180 & 2,10 \\
\hline profilin-1 & PFN1 & 0 & 0,00513 & 1,94 \\
\hline MHC class II antigen & HLA-DRB1 & 0 & 0,00931 & 1,94 \\
\hline rho GDP-dissociation inhibitor & ARHGDIB & 0 & 0,00393 & 1,94 \\
\hline aldolase A & ALDOA & 0,00591 & 0,000974 & $-1,61$ \\
\hline ribosomal protein $\mathrm{L} 7$ & RPL7 & 0,00869 & 0,00143 & $-1,61$ \\
\hline enoyl-CoA hydratase & EHHADH & 0,00250 & 0 & $-1,97$ \\
\hline lactoferrin precursor & LTF & 0,0124 & 0,00154 & $-2,25$ \\
\hline proteasome subunit alpha type- 6 & PSMA6 & 0,0174 & 0,00144 & $-2,52$ \\
\hline Aldo-keto reductase family 1 member $\mathrm{C} 4$ & AKR1C4 & 0,00579 & 0 & $-2,71$ \\
\hline stomatin & STOM & 0,00604 & 0 & $-2,71$ \\
\hline 605 acidic ribosomal protein P0 & RPLPO & 0,00807 & 0 & $-3,20$ \\
\hline phosphoglycerate mutase 1 & PGAM1 & 0,0107 & 0 & $-3,20$ \\
\hline endoplasmic reticulum protein 29 , isoform CRA_b & ERP29 & 0,00894 & 0 & $-3,20$ \\
\hline myeloperoxidase, isoform CRA_d & MPO & 0,0184 & 0,00076 & $-3,48$ \\
\hline L-lactate dehydrogenase $\mathrm{A}$ isoform 1 & LDHA & 0,00859 & 0 & $-3,57$ \\
\hline azurocidin & AZU1 & 0,0161 & 0 & $-3,86$ \\
\hline
\end{tabular}

${ }^{a}$ Normalized Spectral Abundance Factor

${ }^{b} R_{s c}$ is calculated according to semi-quantitative parameter proposed by Old [42] and represents the log ${ }_{2}$ ratio between the protein expression level of control vs the protein expression level of NSCLC tissues. Proteins with $R_{s c} \geq 1,50$ or $\leq-1,50$ were considered differentially expressed

expression status of some protein factors potentially involved in LC development, progression and therapy, and b) the differentially expressed lung proteins between NSCLC and cancer-free tissues in order to define novel biomarkers for NSCLC.

Our data confirmed that ERK1/2, AKT, IKB $\alpha$ and NF$\kappa \beta$ are proteins activated and/or over-expressed in NSCLC. RAS-ERK has, until now, been one of the most extensively studied signaling pathways as ERK1/2 pathway being often up-regulated in different human tumors and therefore represents an attractive target for the development of anticancer drugs. Although the activation status of ERK1/2 has been largely studied in various cell lines, few previous reports have demonstrated an aberrant activation of ERK1/2 in human tumors especially in that of lung $[27,28]$. In this study, we identified an overphosphorylation of ERK1/2 in NSCLC confirming this kinase as a key molecular component implicated also in NSCLC cancer.

PI3K/AKT kinase pathway is another central regulator of cell metabolism, proliferation, and survival [29-33]. Furthermore, AKT is activated in pre-neoplastic and neoplastic lesions and has been linked to ineffectiveness of therapies resulting in poor prognosis [29, 30]. In particular, activation of PI3K/AKT pathway in NSCLC has been associated with increased cellular survival and resistance to chemotherapy and radiation, two important clinical problems encountered in several cancers [29]. Our data showed an over-activation of AKT in NSCLC cancer specimens. We analyzed not only AKT, but also the expression of $\mathrm{IKB} \alpha$ and NF-k $\beta$, other components connected to the PI3K/AKT pathway and involved in the control of survival and inflammation. The overactivation/expression of these molecules strongly supports that ERK1/2 and AKT pathways as well as IKB $\alpha$ / NF- $\mathrm{k} \beta$ axis to be potential biomarkers for diagnosis/ prognosis as well as development of novel targeted drugs in NSCLC. On the other hand, inactivation of NF- $\kappa \beta$, in combination with chemotherapeutic agents, leads to better therapeutic effects in several cancers [31, 32].

Our investigation also focused on the search for novel putative LC biomarkers. Proteomics in cancer research is a technology, by simultaneously examining thousands of proteins, can lead to the discovery of novel biomarkers for cancer [33]. We analyzed and compared the protein expression profile obtained from NSCLC and adjacent cancer-free lung tissues and, through a label-free proteomic approach, defined a set of 20 differentially 
A

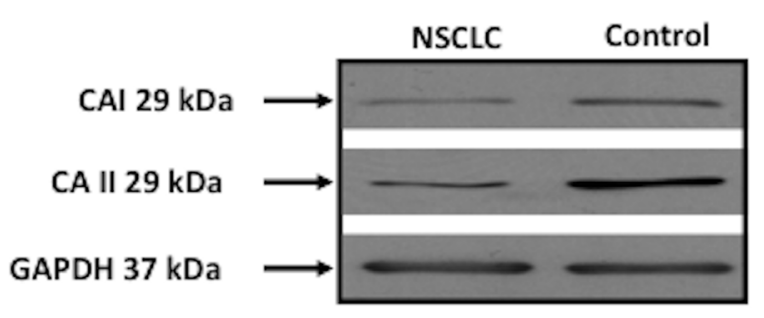

B

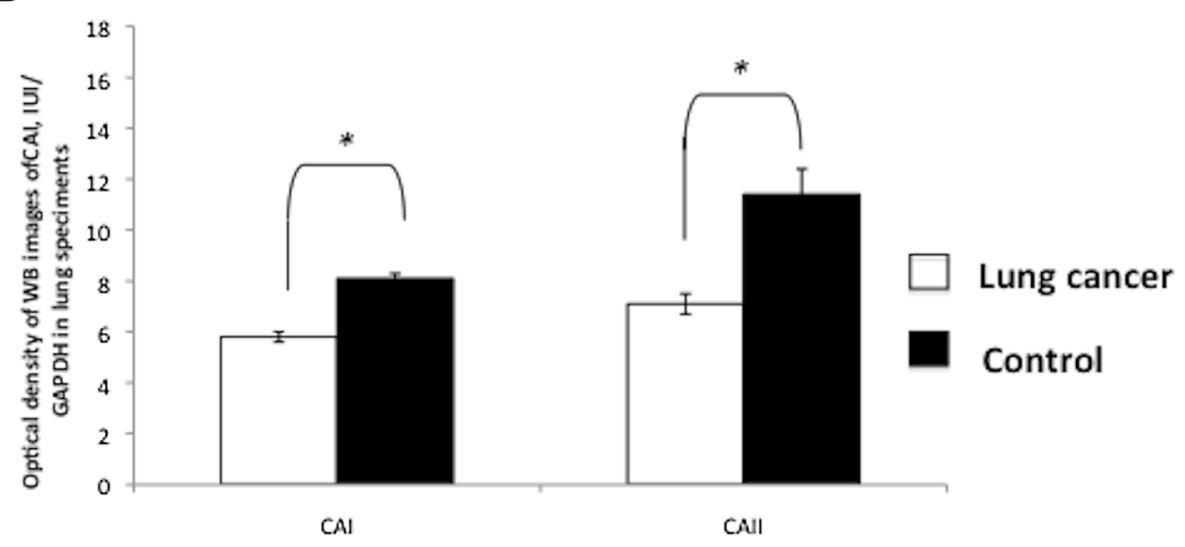

Fig. 4 Western blot analysis confirms CAI and CAll as the two most differentially expressed proteins in NSCLC compared to control tissues. One representative western blot image (a) and graphical representation of pixel quantization (b) of CAl and CAll in lung tissues from 3 NSCLC patients ( 3 with histotype of AC and 1 with histotype of ASC). Each experiment was performed three times in duplicate ${ }^{*}=p<0.05$ by $t$-test analysis. For other details see materials and methods

expressed proteins: seven under-expressed and 13 overexpressed in NSCLC respect to control tissues. To our knowledge, some of them have been previously correlated to LC: aldolase A (ALDOA), phosphoglycerate mutase 1 (PGAM1), l-lactate dehydrogenase A isoform 1 (LDHA), enoyl-CoA hydratase (HADHA), and stomatin (STOM) [33-37]. Here we report the first experimental evidence of a significant increase of the above mentioned proteins in tissues from NSCLC patients. ALDOA, in fact, has been identified as a differentially expressed protein in the bronchoalveolar lavage of patients with LC and/or COPD [33]. Our data of PGAM1 over-expression agrees with that previously found in SCLC and adenocarcinomas (AC) [36]. In addition, LDHA over-expression supports recent findings from NSCLC mouse models both providing a translational impact to murine data and demonstrating the key role of LDHA in LC onset and progression [37]. Regarding the over-expression of HADHA protein, a significant positive correlation between HADHA expression and LC tumor was observed both in cisplatin-resistant LC cells and bioptic specimens from chemotherapy-resistant patients affected by NSCLC, or SCLC or AC has been reported [34]. As for STOM, our observation is in contrast with the only other report describing STOM role in carcinogenesis showing an under-expression of STOM protein n NSCLC tissues with positive lymph node metastasis [35].

The two most under-expressed proteins in NSCLC tissues, CAI and CAII, belong to a widespread family of 16 metallo-isoenzymes that catalyze the interconversion between carbon dioxide and the bicarbonate ion; these enzymes are involved in crucial physiological processes connected to respiration and transport of $\mathrm{CO}_{2} /$ bicarbonate, in $\mathrm{pH}$ and $\mathrm{CO}_{2}$ homeostasis, and in many other metabolic reactions [38]. Furthermore, reduction of CAs levels causes $\mathrm{CO}_{2}$ intracellular retention with consequent increased acidification of extracellular $\mathrm{pH}$, a typical condition observed in rapidly growing tumors $[39,40]$.

Recently, a differential expression of CA isoenzymes has been reported in some malignant tumors, and in particular CAIX has been shown to be prognostic indicator and a potentially important biomarker in the evaluation of cancer [39-41]. CAIX is constitutively upregulated in several cancer types such as lung cancer, breast cancer etc. and therefore, IHC analysis of its expression represents a useful tool for cancer detection, diagnosis and staging in different tissues [41]. CAI and CAII are significantly less expressed in colorectal tumors, rectal carcinomas and pancreatic tumors, but over-expressed in nervous system tumors [40]. Recently, 
a critical role of CAII was reported on LC tumour growth, angiogenesis and survival [41]. To our knowledge, only one study has reported reduction of CAI and CAII expression in LC hypothesizing their role in tumour cell motility, tumour growth and metastasis formation [38]. Our data about CAI and CAII confirm and underline the role of these two isoforms in NSCLC and support a role for them for the first time as biomarkers for disease diagnosis and/or prognosis able to select patients for NSCLC therapy.

\section{Conclusions}

Much effort is being made in the improvement of cancer detection, diagnosis and therapy by identifying more specific molecular biomarkers. In this context, we investigated and confirmed the involvement of ERK1/2, AKT, IKB $\alpha$ and NF- $\kappa \beta$ proteins in NSCLC. Our data supports a role for these proteins as targets for development of novel drugs in LC treatment. Specifically, selective inhibitors of these molecular pathways could have important clinical implications for LC treatment. Comparing protein expression profile between NSCLC and adjacent cancer-free lung tissue, through a label-free proteomic approach, we found 20 differentially expressed proteins; among these we considered and validated CAI and CAII isoforms, the two most under-expressed proteins in cancer tissues. Our data strongly supports a role for these isoenzymes in the diagnosis and/or prognosis of NSCLC disease.

\section{Highlights}

1. ERK1/2, AKT and IKB $\alpha / \mathrm{NF}-\kappa \beta$ pathways are significantly over-activated in NSCLC tissues $v s$ control $[p<0.05]$.

2. Proteomic analysis revealed 20 differently expressed proteins (7 under- and 13 over-expressed) between NSCLC and cancer-free lung tissues $\left(\geq 1.50 \mathrm{R}_{\mathrm{sc}} \leq\right.$ $-1.50)$.

3. Carbonic anhydrase I and II isoforms are strongly over-expressed in NSCLC tissues vs control $(p<0.05)$.

\section{Competing interests}

The authors declare that they have no competing interests.

\section{Authors' contributions}

EN and El have made substantial contributions to conception and design, or acquisition of data, or analysis and interpretation of data; OS, MLM and RP have contributed to experiments execution; $A B$ and $G M$ recruited subjects involved in the study; $A D, A B$ and $S O$ have been involved in drafting the manuscript and revising it critically for important intellectual content; $A D$ has given final approval of the version to be published. All authors read and approved the final manuscript.

\section{Author's information}

E. Nigro and E. Imperlini are the co-first authors.

\section{Acknowledgement}

This work was supported by Grant PON01_02589 (MICROMAP); Grant POR Campania FSE 2007/2013 (CAMPUS-Bioframe) from the Regione Campania, Italy.

\section{Author details}

${ }^{1}$ CEINGE-Biotecnologie Avanzate Scarl, Via G. Salvatore 486, 80145 Naples, Italy. ${ }^{2}$ IRCCS SDN, Via E. Gianturco 113, 80142 Naples, Italy. ${ }^{3}$ Dipartimento di Medicina Molecolare e Biotecnologie Mediche, Università di Napoli Federico II, Via S. Pansini 5, 80131 Naples, Italy. ${ }^{4}$ Dipartimento di Scienze Cardio-Toraciche e Respiratorie, Seconda Università degli Studi di Napoli, Via L. Bianchi, 80131 Naples, Italy. ${ }^{5}$ Dipartimento di Scienze Motorie e del Benessere, Università di Napoli Parthenope, Via Amm. F. Acton 38, 80133 Naples, Italy. ${ }^{6}$ Cattedra di Malattie dell'Apparato Respiratorio, Dipartimento di Medicina e Scienze per la Salute "V Tiberio", Università del Molise, Via De Sanctis, 86100 Campobasso, Italy. ${ }^{7}$ Dipartimento di Scienze e Tecnologie Ambientali Biologiche Farmaceutiche, Seconda Università degli Studi di Napoli, Via G. Vivaldi 42, 81100 Caserta, Italy. ${ }^{8}$ Present address: CEINGE-Biotecnologie Avanzate Scarl, Via G. Salvatore 486, 80145 Naples, Italy.

Received: 5 March 2015 Accepted: 9 June 2015

Published online: 24 June 2015

\section{References}

1. Torre LA, Bray F, Siegel RL, Ferlay J, Lortet-Tieulent J, Jemal A. Global cancer statistics, 2012. CA Cancer J Clin. 2015;65(2):87-108.

2. Sung HJ, Cho JY. Biomarkers for the lung cancer diagnosis and their advances in proteomics. BMB Rep. 2008;41(9):615-25.

3. Pavloua MP, Diamandisa EP. The cancer cell secretome: A good source for discovering biomarkers? J Proteome. 2010;73:1896-906.

4. Frasci G, Lorusso V, Panza N, Comella P, Nicolella G, Bianco A, et al. Gemcitabine plus vinorelbine yields better survival outcome than vinorelbine alone in elderly patients with advanced non-small cell lung cancer. A Southern Italy Cooperative Oncology Group (SICOG) phase III trial. Lung Cancer. 2001;34 Suppl 4:65-9.

5. Piantedosi FV, Caputo F, Mazzarella G, Gilli M, Pontillo A, D'Agostino D, et al. Gemcitabine, ifosfamide and paclitaxel in advanced/metastatic non-small cell lung cancer patients: a phase II study. Cancer Chemother Pharmacol. 2008;61:803-7.

6. Cardarella $\mathrm{S}$, Johnson BE. The impact of genomic changes on treatment of lung cancer. Am J Respir Crit Care Med. 2013;188(7):770-5.

7. Landi MT, Consonni D, Rotunno M, Bergen AW, Goldstein AM, Lubin JH, et al. Environment and Genetics in Lung cancer Etiology [EAGLE] study: An integrative population-based case-control study of lung cancer. BMC Public Health. 2008;8:203-13.

8. Hung RJ, Brennan P, Canzian F, Dabrowska NS, Zaridze D, Lissowska J, et al. Large-Scale Investigation of Base Excision Repair Genetic Polymorphisms and Lung Cancer Risk in a Multicenter Study. J Natl Cancer Inst. 2005;97:567-76.

9. Young RP, Hopkins RJ, Christmas T, Black PN, Metcalf P, Gamble GD. COPD prevalence is increased in lung cancer, independent of age, sex and smoking history. Eur Respir J. 2009:34:380-6.

10. Daniele A, De Rosa A, Nigro E, Scudiero O, Capasso M, Masullo M, et al. Adiponectin oligomerization state and adiponectin receptors airway expression in chronic obstructive pulmonary disease. Int J Biochem Cell Biol. 2012;44(3):563-9.

11. Corbi G, Bianco A, Turchiarelli V, Cellurale M, Fatica F, Daniele A, et al. Potential Mechanisms Linking Atherosclerosis and Increased Cardiovascular Risk in COPD: Focus On Sirtuins. Int J Mol Sci. 2013;14(6):12696-713.

12. Bianco A, Mazzarella G, Turchiarelli V, Nigro E, Corbi G, Scudiero O, et al. Adiponectin: an attractive marker for metabolic disorders in Chronic Obstructive Pulmonary Disease [COPD]. Nutrients. 2013;5(10):4115-25.

13. Nigro E, Scudiero O, Sarnataro D, Mazzarella G, Sofia M, Bianco A, et al. Adiponectin affects lung epithelial A549 cell viability counteracting TNFa and IL-1ß toxicity through AdipoR1. Int J Biochem Cell Biol. 2013;45(6):1145-53.

14. Akcaa H, Demiraya A, Tokguna O, Yokotab J. Invasiveness and anchorage independent growth ability augmented by PTEN inactivation through the PI3KJAKT/NFkB pathway in lung cancer cells. Lung Cancer. 2011;73:302-9.

15. Krysan K, Reckamp KL, Dalwadi H, Sharma S, Rozengurt E, Dohadwala M, et al. Prostaglandin E2 Activates Mitogen-Activated Protein Kinase/Erk Pathway Signaling and Cell Proliferation in Non-Small Cell Lung Cancer 
Cells in an Epidermal Growth Factor Receptor-Independent Manner. Cancer Res. 2005;65:6275-81.

16. Stinchcombea TE, Johnsonb GL. MEK inhibition in non-small cell lung cancer. Lung Cancer. 2014;86:121-5.

17. Viatour P, Merville MP, Bours V, Chariot A. Phosphorylation of NF-kB and IkB proteins: implications in cancer and inflammation. Trends Biochem Sci. 2005;3:43-52.

18. Zer A, Leighl N. Promising Targets and Current Clinical Trials in Metastatic Non-Squamous NSCLC. Front Oncol. 2014;25(4):329.

19. Steelman LS, Chappell WH, Abrams SL, Kempf RC, Long J, Laidler P, et al. Roles of the Raf/MEK/ERK and PI3K/PTEN/Akt/mTOR pathways in controlling growth and sensitivity to therapy-implications for cancer and aging. Aging (Albany NY). 2011;3(3):192-222.

20. Brunese L, Greco B, Setola FR, Lassandro F, Guarracino MR, De Rimini M, et al. Non-small cell lung cancer evaluated with quantitative contrastenhanced CT and PET-CT: net enhancement and standardized uptake values are related to tumour size and histology. Med Sci Monit. 2013;7(19):95-101.

21. Guo S, Yan F, Xu J, Bao Y, Zhu J, Wang X, et al. Identification and validation of the methylation biomarkers of non-small cell lung cancer [NSCLC]. Clin Epigenetics. 2015;7(1):3.

22. Planque C, Kulasingam V, Smith CR, Reckamp K, Goodglick L, Diamandis EP. Identification of Five Candidate Lung Cancer Biomarkers by Proteomics Analysis of Conditioned Media of Four Lung Cancer Cell Lines. Mol Cell Proteomics. 2009:8:2746-58.

23. Daniele A, Cammarata R, Masullo M, Nerone G, Finamore F, D'Andrea M, et al. Analysis of adiponectin gene and comparison of its expression in two different pig breeds. Obesity (Silver Spring). 2008;16(8):1869-74.

24. Imperlini E, Colavita I, Caterino M, Mirabelli P, Pagnozzi D, Del Vecchio L, et al. The secretome signature of colon cancer cell lines. J Cell Biochem. 2013;114:2577-87.

25. Imperlini E, Orrù S, Corbo C, Daniele A, Salvatore F. Altered brain protein expression profiles are associated with molecular neurological dysfunction in the PKU mouse model. J Neurochem. 2014;129(6):1002-12.

26. Spaziani S, Imperlini E, Mancini A, Caterino M, Buono P, Orrù S. Insulin-like growth factor 1 receptor signaling induced by supraphysiological doses of IGF-1 in human peripheral blood lymphocytes. Proteomics. 2014;14:1623-9.

27. Vicent S, López-Picazo JM, Toledo G, Lozano MD, Torre W, Garcia-Corchón $C$, et al. ERK $1 / 2$ is activated in non-small-cell lung cancer and associated with advanced tumours. Br J Cancer. 2004;8:1047-52.

28. Adjei AA. The Role of Mitogen-Activated ERK-Kinase Inhibitors in Lung Cancer Therapy. Clin Lung Cancer. 2005;7:221-3.

29. Holland WS, Chinn DC. Lara, Jr PN. Mack PC. Effects of AKT inhibition on HGF-mediated erlotinib resistance in non-small cell lung cancer cell lines. J Cancer Res Clin Oncol: Gandara DR; 2014.

30. Altomare DA, Testa JR. Perturbations of the AKT signaling pathway in human cancer. Oncogene. 2005;14:7455-64.

31. Van Waes C. Nuclear factor-kappaB in development, prevention, and therapy of cancer. Clin Cancer Res. 2007;13(4):1076-82.

32. Choi KE, Hwang CJ, Gu SM, Park MH, Kim JH, Park JH, et al. Cancer cell growth inhibitory effect of bee venom via increase of death receptor 3 expression and inactivation of NF-kappa B in NSCLC cells. Toxins [Basel]. 2014;6(8):2210-28.

33. Pastor MD, Nogal A, Molina-Pinelo S, Meléndez R, Salinas A, González De la Peña $M$, et al. Identification of proteomic signatures associated with lung cancer and COPD. J Proteomics. 2013;89:227-37.

34. Kageyama T, Nagashio R, Ryuge S, Matsumoto T, lyoda A, Satoh Y, et al. HADHA is a potential predictor of response to platinum-based chemotherapy for lung cancer. Asian Pac J Cancer Prev. 2011;12(12):3457-63.

35. Arkhipova KA, Sheyderman AN, Laktionov KK, Mochalnikova W, Zborovskaya IB. Simultaneous expression of flotillin-1, flotillin-2, stomatin and caveolin-1 in non-small cell lung cancer and soft tissue sarcomas. BMC Cancer. 2014;14:100.

36. Bührens Rl, Amelung JT, Reymond MA, Beshay M. Protein expression in human non-small cell lung cancer: a systematic database. Pathobiology. 2009;76(6):277-85.

37. Xie H, Hanai J, Ren JG, Kats L, Burgess K, Bhargava P, et al. Targeting lactate dehydrogenase-a inhibits tumorigenesis and tumor progression in mouse models of lung cancer and impacts tumor-initiating cells. Cell Metab. 2014;19(5):795-809.

38. Chianga WL, Chuc SC, Yangd SS, Lie MC, Laif JC, Yangb SF, et al. The aberrant expression of cytosolic carbonic anhydrase and its clinical significance in human non-small cell lung cancer. Cancer Lett. 2002;188:199-205.

39. Takakura M, Yokomizo A, Tanaka Y, Kobayashi M, Jung G, Banno M, et al. Carbonic Anhydrase I as a New Plasma Biomarker for Prostate Cancer. ISRN Oncology Volume. 2012;2012:768190.

40. Sheng W, Dong M, Zhou J, Li X, Dong Q. Down Regulation of CAll is Associated With Tumor Differentiation and Poor Prognosis in Patients With Pancreatic Cancer. J Surg Oncol. 2013;107:536-43.

41. Zhou Y, Mokhtari RB, Pan J, Cutz E, Yeger H. Carbonic Anhydrase II Mediates Malignant Behavior of Pulmonary Neuroendocrine Tumors. Am J Respir Cell Mol Biol. 2014;52(2):183-92.

42. Old WM, Meyer-Arendt K, Aveline-Wolf L, Pierce KG, Mendoza A, Sevinsky JR, et al. Comparison of label-free methods for quantifying human proteins by shotgun proteomics. Mol Cell Proteomics. 2005;4(10):1487-502.

\section{Submit your next manuscript to BioMed Central and take full advantage of:}

- Convenient online submission

- Thorough peer review

- No space constraints or color figure charges

- Immediate publication on acceptance

- Inclusion in PubMed, CAS, Scopus and Google Scholar

- Research which is freely available for redistribution 